\title{
Erratum: Alfimeprase to succeed Genentech's alteplase?
}

Brian Vastag

Nat. Biotechnol. 24, 875-876 (2006)

In the 6th paragraph, the statement "in 1996, Genentech launched Alteplase" is incorrect. Genentech received FDA approval of Alteplase for heart attack in 1987. In 1996, it received approval for Alteplase's second indication, stroke.

\section{Erratum: Diversifying chemical arrays}

\section{Laura DeFrancesco}

Nat. Biotechnol. 24, 799 (2006)

In the print version of the article, the author of the featured article is incorrectly identified as Brandord et al. The author's name is Bradner.

\section{Corrigendum: All in the RNA family}

Beverly L. Davidson

Nat. Biotechnol. 24, 951-952 (2006)

In the fifth paragraph, the abbreviation for prostate-specific membrane antigen (PSMA) was mistakenly written several times as PMSA. This error also appears in Figure 1.

\section{Corrigendum: Engineering and characterization of a superfolder green} fluorescent protein

Jean-Denis Pédelacq, Stéphanie Cabantous, Timothy Tran, Thomas C Terwilliger \& Geoffrey S Waldo

Nat. Biotechnol. 24, 79-88 (2005)

In the legend for Figure $4 \mathrm{~b}$ and in the last line of paragraph 6 in Methods, "number of moles" should be moles. Also in Methods, paragraph 3 , "superfolder GFP (27.747 kDa/mole)..." should read "superfolder GFP (27747 g/mole)" and "folding reporter GFP (27.742 kDa/mole)..." should read “...folding reporter GFP (27,742 g/mole)." The error has been corrected in the PDF version of the article.

\section{Retraction: Identification of genes that function in the TNF- $\alpha$-mediated} apoptotic pathway using randomized hybrid ribozyme libraries

Hiroaki Kawasaki, Reiko Onuki, Eigo Suyama \& Kazunari Taira

Nat. Biotechnol. 20, 376-380 (2002)

Although the gene discovery technology described in this paper has been demonstrated to have practical utility by several independent researchers, the first author of the paper failed to maintain a proper data notebook to support the results presented. As this constitutes nonadherence to the ethical standards in scientific research, and in accordance with the recommendations from the National Institute of Advanced Industrial Science \& Technology (AIST), R. Onuki, E. Suyami and K. Taira respectfully retract this paper. H. Kawasaki declines to associate himself with this retraction and maintains that all the data contained in the paper are valid. 